\title{
Glycine Receptors in the Nervous Tissue and Their Functional Role
}

\author{
V. N. Nikandrov ${ }^{a}$ and T. V. Balashevich ${ }^{b, 1}$ \\ ${ }^{a}$ Polesskii State University, Pinsk, Belarus \\ ${ }^{b}$ Institute of Physiology of the National Academy of Sciences of Belarus, ul. Academicheskaya 28, Minsk, 220072 Belarus \\ tel.: (+375 29) 767-97-30; (+375 29) 149-54-36; e-mail: tbalashevich@bk.ru \\ Received October 25, 2012
}

\begin{abstract}
The literature data on glycine metabolism in neural tissue, the mitochondrial Gly-cleaving system, and the Gly uptake system in neural and glial cells are summarized. Brief description of localization and abundance of glycine receptors and specific binding sites in the mammalian nervous tissue is given. Four types of glycine binding receptors are described. These include own specific glycine receptor (GlyR), ionotropic glutamate receptor, which selectively binds $\mathrm{N}$-methyl-D-aspartate selectively (NMDAR), and ionotropic receptors of $\gamma$-aminobutyrate $\left(\mathrm{GABA}_{\mathrm{A}} \mathrm{R}, \mathrm{GABA}_{\mathrm{C}} \mathrm{R}\right)$.
\end{abstract}

Keywords: glycine, metabolism, receptors, neuroprotective action, nervous tissue cells, regulatory effects

DOI: $10.1134 / \mathrm{S} 1990750813030104$

\section{INTRODUCTION}

Glycine (Gly) exhibits various vitally important functions in the body [1-9]. It was found in various proteins and biologically active proteins; it is an immediate precursor for synthesis of the heme porphyrin moiety and a component for synthesis of purine bases [10]. This amino acid is also involved in regulation of neurotransmitter synaptic transmission and the central and peripheral nervous system [1, 7]. Gly receptors localized in various regions of the brain and spinal cord mediated the inhibitory action on neurons and thus reduce release of excitatory amino acids [1-3]. Gly also acts as a co-agonist of $\gamma$-amino butyrate receptor (GABAR) and glutamate NMDA receptor (NMDAR) [4-8].

Although Gly-based pharmacological preparations demonstrate a wide range of positive effects (e.g., improvement of memory and associative processes, the antidepressant action, reduced feelings of anxiety, fear, psychoemotional stress, etc.), mechanisms responsible for their realization still require better understanding and in most even the best studied links of these mechanisms are based on numerous hypothesis rather than well-documented facts.

Possible Gly effects on viability of cultured nervous tissue cells remain poorly investigated as most studies in this field do investigate bioelectric activity of cell membranes influenced by Gly as a neurotransmitter $[7-9,11,12]$.

However, it has been recently demonstrated that Gly in the range of concentrations from 0.01 to $25.0 \mathrm{mM}$ attenuates degenerative processes in the spi-

\footnotetext{
${ }^{1}$ To whom correspondence should be addressed.
}

nal cord cell layer and C6 glioma induced by serum (trophic factor) deprivation [13-20]. During the whole period of observation $(72 \mathrm{~h})$ decreased activity of 2-calpain (EC 3.4.22.53), lactate dehydrogenase (EC 1.1.1.27) was registered in cells and conditioned media of these cultures; this was also accompanied by decreased cell death and maintenance of proliferative activity in the nerve tissue cells [13-19]. Gly addition also caused a 2-7-fold increase in spinal cord cell survival [20]. Under conditions of prolonged serum deprivation in the cultural medium $0.1 \mathrm{mM}$ Gly exhibited the mitogenic effect on rat spinal cord cells (14 days in vitro): there was an increase in cell DNA content and appearance of young neurons in the culture $[13,16]$.

It was found that Gly critically influenced plasminogen effects. For example, 0.01 or $0.1 \mathrm{mM}$ Gly added together with plasminogen $\left(10^{-7} \mathrm{M}\right)$ potentiated the protector effect of this zymogen on C6 and spinal cord cells; in the presence of $10^{-8} \mathrm{M}$ plasminogen Gly increased the stimulating effect of this zymogen on C6 glioma cells. The stabilizing action was observed at both morpho-functional and metabolic effects [14, $17,20-22]$.

These numerous Gly effects on the nervous tissue in vitro suggest a very important role of this amino acid for regulation of viability of nervous tissue cells (particularly, changes in cell cycle, cell protection against various damaging factors, principal changes in the mode of action of proteins exhibiting neurotrophic (plasminogen-like) properties on target cells. Detailed elucidation of their mechanisms will clearly open perspectives for subsequent multidisciplinary studies. 


\section{GLYCINE METABOLISM} IN THE NERVOUS TISSUE

Although glycine has been identified in proteins more than 200 years ago, studies on its dynamic content in various brain structures still cause scientific debates [23, 24].

Brain content of Gly (and GABA as well) prevails (in the reducing order) in such structures as midbrain, thalamus, cerebellum, temporal cortex, and corpus collosum [24]. It is generally accepted that the brain content of free Gly is normally $1.3 \mu \mathrm{M}$ and in the cerebrospinal fluid it is two orders of magnitude less. Since Gly consumption in the nervous tissue is rather high and its supply from blood occurs rather slowly, the brain synthesizes a significant proportion of Gly de novo [25].

Glucose and serine are the major sources for Gly biosynthesis in the central nervous system (CNS) [24]. Serine may be formed from glucose via 3-phosphoglycerate formation; in addition, it is readily penetrates from blood circulation into the brain across the blood brain barrier. In the nervous tissue Gly synthesis de novo involves serine hydroxymethyltransferase (EC 2.1.2.1) [25]. High activity of this enzyme was found in metabolic pools of the brain and spinal cord, for example, in the cerebellum and the anterior horn of the spinal cored. Serine hydroxymethyltransferase activity correlated first of all with glycine content in these structures [2].

Two other sources for Gly synthesis in the human and animal nervous systems include glutamate and glyoxylate [24]. Since brain glutamate concentration (about $10.6 \mu \mathrm{M}$ ) is the highest among amino acids, while glyoxylate concentrations are insignificant, glutamate makes the major contribution to Gly formation. Glutamine, a precursor of both excitatory (Glu, Asp) and inhibitory (GABA, Gly) amino acid neurotransmitters enters neurons from the glia [26].

At least three pathways of Gly catabolism do exist in the nervous tissue [24]. The first one is based on reversibility of the reaction of Ser conversion into Gly in the brain tissue and therefore serine hydroxymethyltransferase may act as the Gly metabolizing enzyme $[24,27]$. The second pathway involves oxidases of amino acids (EC 1.4.3.2, 1.4.3.3); in the CNS these highly abundant enzymes use Gly and other amino acids as substrates [24, 27, 28]. The third system of Gly cleavage is localized only in mitochondria and is atypical amino acid decarboxylase as its activity depends on both $\mathrm{NAD}^{+}$and tetrahydrofolate (THF) [24]. The Gly cleavage system catalyzes Gly cleavage into methylenetetrahydrofolate, carbon dioxide, and ammonia; methylene-THF undergoes further oxidation with carbon dioxide formation, the end product of this amino acid [24].

The Gly cleavage systems were found not only in neuronal cells, but also in astrocytes [29-31]. This is consistent with abundance of metabolic reactions in the nervous tissue in accordance with functional features of cell types.

The brain mitochondrial Gly cleavage system includes four components: P-protein (pyridoxal phosphate-dependent glycine decarboxylase), T-protein (THF-dependent aminomethyl transferase), H-protein (containing lipoic acid as proton carrier), and L-protein (lipoamide dehydrogenase) [29-31].

In situ hybridization analysis has shown that protein P mRNA is prefentially expressed in glia-like cells (including Bergmann glia in the cerebellum), while Tand $\mathrm{H}$-protein mRNAs are registered in neurons and glia-like cells [29].

Only expression of $\mathrm{T}$ - and $\mathrm{H}$-protein mRNAs was found in the spinal cord; the primary culture of spinal cord astrocytes contained only the proton carrier (H-protein) lacking any enzymatic activity. It should be noted that the activity of the mitochondrial Gly cleavage system was higher in the primary culture of rat brain cortex astrocytes than in hepatocytes [29].

In the spinal cord and the brainstem Gly functions as an inhibitory neurotransmitter, while in the hippocampus, brain cortex, cerebellum, and the olfactory bulb it acts as the stimulating modulator of NMDAR [29]. Analysis of literature data suggests that the Gly cleavage system is involved in pathogenesis of nonketotic hyperglycemia via modulation of NMDAR in the anterior brain and the cerebellum.

Consequently, Gly metabolism includes various catabolic and anabolic processes, which have several intersecting (cross-talk) points, and may be summarized into the scheme of Gly metabolism (Fig. 1). These cross-talks allow one processes to prevail over the other ones. For example, in rat brain astricytes the activity of the mitochondrial Gly cleavage system in higher than the activity of enzymes catalyzing reverse conversion of Gly into serine and therefore probability of Gly cleavage in astrocytes is higher than its use for serine formation [31].

The spinal cord has high and low affinity systems of Gly uptake $\left(K_{\mathrm{m}}<0.05 \mathrm{mM}\right.$ and $K_{\mathrm{m}}>0.1 \mathrm{mM}$, respectively), while the brain cortex has only the low affinity uptake system [24].

It is generally believed that in the brain Gly is preferentially involved in activation of the glutamate receptor [32,33]: activation of NMDAR requires agonist concentrations of about $0.0001-0.1 \mathrm{mM}$. In this case it is reasonable to suggest poor (if any) contribution of the low affinity uptake system of the brain into regulation of cortical cell functioning.

However, it should be also noted that brain Gly concentrations were evaluated in a pool (mass) of the tissue or the brain cortex. The latter cannot rule out that at certain time interval and in certain sites physiological concentrations of this amino acid may be reasonably high for involvement of the low affinity uptake system into physiological functioning of Gly. This problem clearly needs further investigation. 